\title{
Drought and host selection influence bacterial community dynamics in the grass root microbiome
}

\author{
Dan Naylor ${ }^{1,2}$, Stephanie DeGraaf ${ }^{3}$, Elizabeth Purdom ${ }^{3}$ and Devin Coleman-Derr ${ }^{1,2}$ \\ ${ }^{1}$ Department of Plant and Microbial Biology, 111 Koshland Hall, University of California, Berkeley, CA, USA; \\ ${ }^{2}$ Plant Gene Expression Center, UC Berkeley, USDA-ARS, Albany, CA, USA and ${ }^{3}$ Department of Statistics, \\ University of California, Berkeley, CA, USA
}

\begin{abstract}
Root endophytes have been shown to have important roles in determining host fitness under periods of drought stress, and yet the effect of drought on the broader root endosphere bacterial community remains largely uncharacterized. In this study, we present phylogenetic profiles of bacterial communities associated with drought-treated root and rhizosphere tissues of 18 species of plants with varying degrees of drought tolerance belonging to the Poaceae family, including important crop plants. Through 16S rRNA gene profiling across two distinct watering regimes and two developmental time points, we demonstrate that there is a strong correlation between host phylogenetic distance and the microbiome dissimilarity within root tissues, and that drought weakens this correlation by inducing conserved shifts in bacterial community composition. We identify a significant enrichment in a wide variety of Actinobacteria during drought within the roots of all hosts, and demonstrate that this enrichment is higher within the root than it is in the surrounding environments. Furthermore, we show that this observed enrichment is the result of an absolute increase in Actinobacterial abundance and that previously hypothesized mechanisms for observed enrichments in Actinobacteria in drought-treated soils are unlikely to fully account for the phenomena observed here within the plant root.
\end{abstract}

The ISME Journal (2017) 11, 2691-2704; doi:10.1038/ismej.2017.118; published online 28 July 2017

\section{Introduction}

Few environmental stresses are as omnipresent and devastating to agriculture as drought, which annually results in billions of dollars in losses worldwide (Lesk et al., 2016). While some crop responses to drought are conserved across most species, including increased ABA production and stomatal closure, other adaptations are host-specific, such as special carbon uptake pathways, increased cuticle thickness and altered root morphology (Fang and Xiong, 2015). As plants are intimately intertwined with the communities of microbes living in and around them (Gaiero et al., 2013; Philippot et al., 2013; Berg et al., 2016), perturbations in plant physiology and metabolism, such as those resulting from drought response mechanisms, can be expected to alter the composition of the plant microbiome with potential consequences for host fitness (Berg et al., 2014). However, little information is available on how drought influences the plant root microbiome, and

Correspondence: D Coleman-Derr, Plant Gene Expression Center, UC Berkeley, USDA-ARS, 800 Buchanan Street, Albany, CA 94710, USA.

E-mail: colemanderr@berkeley.edu

Received 3 February 2017; revised 20 April 2017; accepted 7 June 2017; published online 28 July 2017 the degree to which such changes are conserved across different plant hosts.

Recent studies have noted enrichment of the bacterial taxa Actinobacteria in drought-treated soils across a range of environments (Bouskill et al., 2013, 2016) and in drought-treated rhizospheres (soils adhering to the root surface) for several plant species (Nessner Kavamura et al., 2013; Taketani et al., 2016). It has been suggested that this observed relative enrichment is due to differing life strategies of soil microorganisms; specifically, the spore-forming ability of Actinobacteria, which allows them to enter a stable and quiescent state during periods of environmental stress, would lead them to persist under drought conditions, while less fit bacterial lineages decrease in abundance (Nessner Kavamura et al., 2013; Taketani et al., 2016). Whether Actinobacteria are also enriched within the plant root under drought, and the degree to which such enrichment patterns are generalizable across a broad range of hosts and Actinobacterial taxa, remains unknown. We hypothesize that the plant root microbiome will exhibit significant shifts during drought, including an increase in Actinobacteria, and that these changes will be, at least in part, host specific as a result of differing drought response mechanisms. 
While the effect of drought on the root microbiome remains largely unexplored, more effort has been made to understand the role of plant host genotype in shaping root-associated bacterial communities (Kuske et al., 2002; Brusetti et al., 2005; Aleklett et al., 2015; Bulgarelli et al., 2015). Different host species not only have unique exudation patterns (Cavaglieri et al., 2009) but also root architecture, lifespan and rooting depth, all of which have been shown to influence microbiome structure (Fierer et al., 2003; Shi et al., 2011; Berendsen et al., 2012; Badri et al., 2013; Chaparro et al., 2013). As these root phenotypes are under genetic control of their hosts, genetically similar hosts might be expected to harbor comparable microbiome profiles (Parker and Spoerke, 1998; Ushio et al., 2008). Indeed, host phylogenetic relationships were recently shown to be correlated with microbiome dissimilarity in the rhizosphere (Bouffaud et al., 2014), and among accessions of a single species, similar hosts had more comparable microbiota in rhizosphere and roots (Peiffer et al., 2013; Bulgarelli et al., 2015). However, the relative strength of this correlation within roots and rhizospheres have yet to be compared within a phylogenetically diverse host framework. Additionally, it remains to be seen whether drought stress strengthens or weakens the correlation between host phylogeny and bacterial community composition. We hypothesize that a positive correlation between host phylogenetic and microbiome distance exists and will be strongest inside the root, where plants have greater influence over bacterial interactions and recruitment; furthermore, we hypothesize that drought will strengthen this correlation, because the metabolic and phenotypic factors driving host-specific differences in bacterial recruitment are, at least in part, adaptive traits to differing water availability in each host's native environment.

To test these hypotheses, we analyzed bacterial community composition in root endosphere and rhizosphere for 18 grass species in the Poaceae clade, grown in the field under drought conditions. We included nine species with $\mathrm{C} 4$ carbon metabolism and nine with C3 carbon metabolism, as plant species using C4 metabolism are better adapted to arid environments (Fang and Xiong, 2015). The grasses we chose included agronomically valuable C3 crops with moderate to poor drought tolerance, such as wheat and barley (Nezhadahmadi et al., 2013; Ghotbi-Ravandi et al., 2014), and also highly drought-tolerant C4 species, such as sorghum (Sabadin et al., 2012). Our experimental design allowed us to investigate the effect of drought on the grass microbiome, to observe whether such effects are unique or conserved across a broad range of hosts with differing drought tolerances, and assess the degree of correlation between host phylogeny and the root microbiome under normal and drought conditions.

\section{Materials and methods}

\section{Experimental design}

The primary experimental field used in our study is an agricultural field site located in Albany, California $\left(37.8864^{\circ} \mathrm{N}, 122.2982^{\circ} \mathrm{W}\right)$ characterized by a silty loam soil with low $\mathrm{pH}$ (5.2) (Supplementary Table S1). A smaller experiment at an additional field site was conducted to test the reproducibility of results observed at the first site (see Supplementary Information). The second site is located at the Kearney Agricultural Research station $\left(36.6008^{\circ} \mathrm{N}\right.$, $119.5109^{\circ} \mathrm{W}$ ), run by the University of California, and has sandy loam soils with a silty substratum with neutral $\mathrm{pH}$ (7.37) (Supplementary Table S1). The nine C3 and nine C4 grasses and tomato germplasm used in the primary study are described in Supplementary Table S2, and include nine C3 species (two varieties of Triticum aestivum (TaB and $\mathrm{TaG})$, Triticum monococcum (Tm), Triticum turgidum (Tt), Secale cereale (Sc), Hordeum vulgare (Hv), Avena sativa (As), Festuca arundinaceae (Fa), Brachypodium distachyon (Bd)), nine C4 species (two varieties of Sorghum bicolor ( $\mathrm{SbA}$ and $\mathrm{SbH}$ ), Sorghum laxiflorum (Sl), Sorghastrum nutans (Sn), Miscanthus sinensis (Ms), Bothriochloa bladhii (Bb), Zea mays $(\mathrm{Zm})$, Pennisetum americanum (Pa), Eragrostis tef (Et)) and an outgroup, Solanum lycopersicum (Out). Seeds were surface sterilized, germinated and grown in growth chambers for 2 weeks as described in the Supplementary Information, after which all plants were transplanted to the field in five full factorial replicate blocks. Under control conditions, plants were watered for $1 \mathrm{~h}$ three times per week using drip irrigation with $1.89 \mathrm{~L} \mathrm{~h}^{-1}$ rate flow emitters under control treatment, and no water was given during drought treatments. For the early (preflowering) time point, we imposed drought 2 weeks post-transplantation and harvested 5 weeks post-transplantation. For the late (postflowering) time point, we imposed drought 4 weeks posttransplantation, and harvested at 12 weeks posttransplantation. At collection time, the percentage of plants that had transitioned from vegetative to reproductive growth was $2.1 \%$ at the early time point (almost entirely attributable to Solanum lycopersicum), in contrast to $93.4 \%$ at the late time point (Miscanthus sinensis was the only plant that had not flowered). For soil moisture content and plant biomass, samples were collected at harvest time in all five replicate blocks. For microbiome data, bulk soil, rhizosphere and root samples were collected from three replicate blocks (due to limitations in time and funding available, only three of the five replicate blocks were used for microbiome analyses) and genomic DNA prepared as in Coleman-Derr et al. (2016) with minor modifications, for 206, 207 and 211 soil, root and rhizosphere samples. In brief, $15 \mathrm{~cm}$ soil core samplers were used to collect bulk soil up to $\sim 20 \mathrm{~cm}$ from the base of the plant. Root systems were excavated from the top $25 \mathrm{~cm}$ of soil, 
shaken briefly to remove excess soil and root tissue was collected and stored in epiphyte removal buffer $\left(0.75 \% \mathrm{KH}_{2} \mathrm{PO}_{4}, 0.95 \% \mathrm{~K}_{2} \mathrm{HPO}_{4}, 1 \%\right.$ Triton X-100 in $\mathrm{dd}_{2} \mathrm{O}$; filter sterilized at $0.2 \mu \mathrm{M}$ ) on ice. Root samples were then sonicated for $10 \mathrm{~min}$ at 'Low' in a Diagenode Bioruptor (Diagenode Inc., Denville, NJ, USA); residual soil remaining at the bottom of the tube after this step was retained as 'rhizosphere', whereas the roots were transferred to a new tube, rinsed two times with sterile water and stored in sterile epiphyte removal buffer.

\section{Plant phylogenetic distances}

Phylogenetic distances between our 19 hosts were determined through the analysis of three genes $r b c L$ (Rubisco large subunit), $n d h F$ (NADH dehydrogenase subunit F) and matK (tRNA-Lys) intron; the primers used for the amplification of these regions are described in Grass Phylogeny Working Group II (2012). Genomic DNA extractions, PCR and Sanger sequencing were carried out as described in the Supplementary Information.

\section{Amplicon data}

Samples from the primary data set were amplified using a dual-indexed $16 \mathrm{~S}$ rRNA Illumina iTags primer set specific to the $\mathrm{V} 3-\mathrm{V} 4$ region $(341 \mathrm{~F}$, 5'-CCTACGGGNBGCASCAG-3' and 785 R, 5'-GAC TACNVGGGTATCTAATCC-3') as described in Takahashi et al. (2014) using reaction conditions as described in Coleman-Derr et al. (2016). Raw reads were trimmed, quality-filtered, chimerachecked, clustered and assigned taxonomies as in Coleman-Derr et al. (2016), yielding 2760 highabundance operational taxonomic units (OTUs) after removing OTUs with $<5$ reads in at least five samples and then normalizing to 10000 reads per sample. Additional sequence data sets generated for samples from our secondary field site, for a comparison of DNA extraction methods and for the fungal ITS2 sequencing, are described in the Supplementary Information. Raw sequencing data were deposited in the NCBI Short Read Archive, BioProjectID PRJNA369551, accession number SRP098753.

\section{Actinobacterial-specific ddPCR}

Digital droplet PCR using Bio-Rad QX200 Droplet Reader (Bio-Rad, Hercules, CA, USA) was performed according to the manufacturer's instructions. The reaction volume was $20 \mu \mathrm{l}$, including $900 \mathrm{~nm}$ Actinobacteria-specific primers (Act920F3/ Act1200R (Bacchetti De Gregoris et al., 2011) and Act664F/Act941R (Yang et al., 2015)), 1x EvaGreen supermix and $1 \mathrm{ng}$ template DNA. Droplets were generated using $20 \mu \mathrm{l}$ sample and $70 \mu \mathrm{l}$ oil; $30 \mu \mathrm{l}$ droplets were used for amplification, with the following conditions: $5 \mathrm{~min}$ at $95^{\circ} \mathrm{C}$, then 40 cycles of $30 \mathrm{~s}$ at $95^{\circ} \mathrm{C}, 1 \mathrm{~min}$ at $61.5^{\circ} \mathrm{C}$, followed by $5 \mathrm{~min}$ at $4{ }^{\circ} \mathrm{C}, 5 \mathrm{~min}$ at $90^{\circ} \mathrm{C}$ and holding at $4{ }^{\circ} \mathrm{C}$. All steps were performed with a slow ramp rate of $2^{\circ} \mathrm{Cs}^{-1}$. Plates were promptly analyzed after conclusion of PCR runs using the aforementioned droplet reader. The Actinobacteria-specific primers used here have reported specificities of 91.8 and $99.4 \%$, respectively (Bacchetti De Gregoris et al., 2011; Yang et al., 2015), and in silico analyses using SILVA TestPrime (Quast et al., 2013) revealed similar specificity values of 87.8 and $99.3 \%$.

\section{Statistical analyses}

All statistical analyses were performed in $\mathrm{R}$ using the normalized reduced data set with 2760 OTUs and 612 samples, and descriptions of individual analyses and use of specific packages is described in detail in the Supplementary Information.

\section{Results}

We planted 18 grass lineages (Supplementary Table S2) including varieties of wheat, rye, barley, oat, Brachypodium, tall fescue, sorghum, Indian grass, Miscanthus, plume grass, maize, millet and tef, as well as one dicot species, tomato, as an outgroup. Plants were grown in five replicated randomized blocks (Supplementary Figure S1) within our experimental field. As plant developmental stage has been implicated as a factor shaping the plant microbiome (Edwards et al., 2015; Dombrowski et al., 2017), and as drought has different effects on host physiology depending on when it occurs in development (Fang and Xiong, 2015), we used two distinct treatments for drought-treated replicates. In half of each of the five full factorial replicate blocks, we imposed drought for non-control plants at week 4 and harvested 3 weeks later before most plants had flowered; in the other half of the five blocks, we imposed drought for non-control plants at week 6 and harvested 8 weeks later after the majority of plants had flowered. This design allowed us to obtain samples from both preflowering (early) and postflowering (late) time points. To test whether irrigation treatments had a significant effect on both plant growth and available soil moisture, all samples in the five replicate blocks belonging to the late time point were used to measure above-ground shoot biomass and soil water content. Both biomass and soil moisture were significantly lower under drought (Supplementary Figure S2), suggesting both environment and plant were affected, even across the broad range of hosts.

To obtain microbiome data, all samples from three replicate blocks, including both early and late time points, were harvested and bacterial communities in root, rhizosphere and bulk soil samples were examined. We chose to focus on bacterial communities because of their demonstrated potential for 

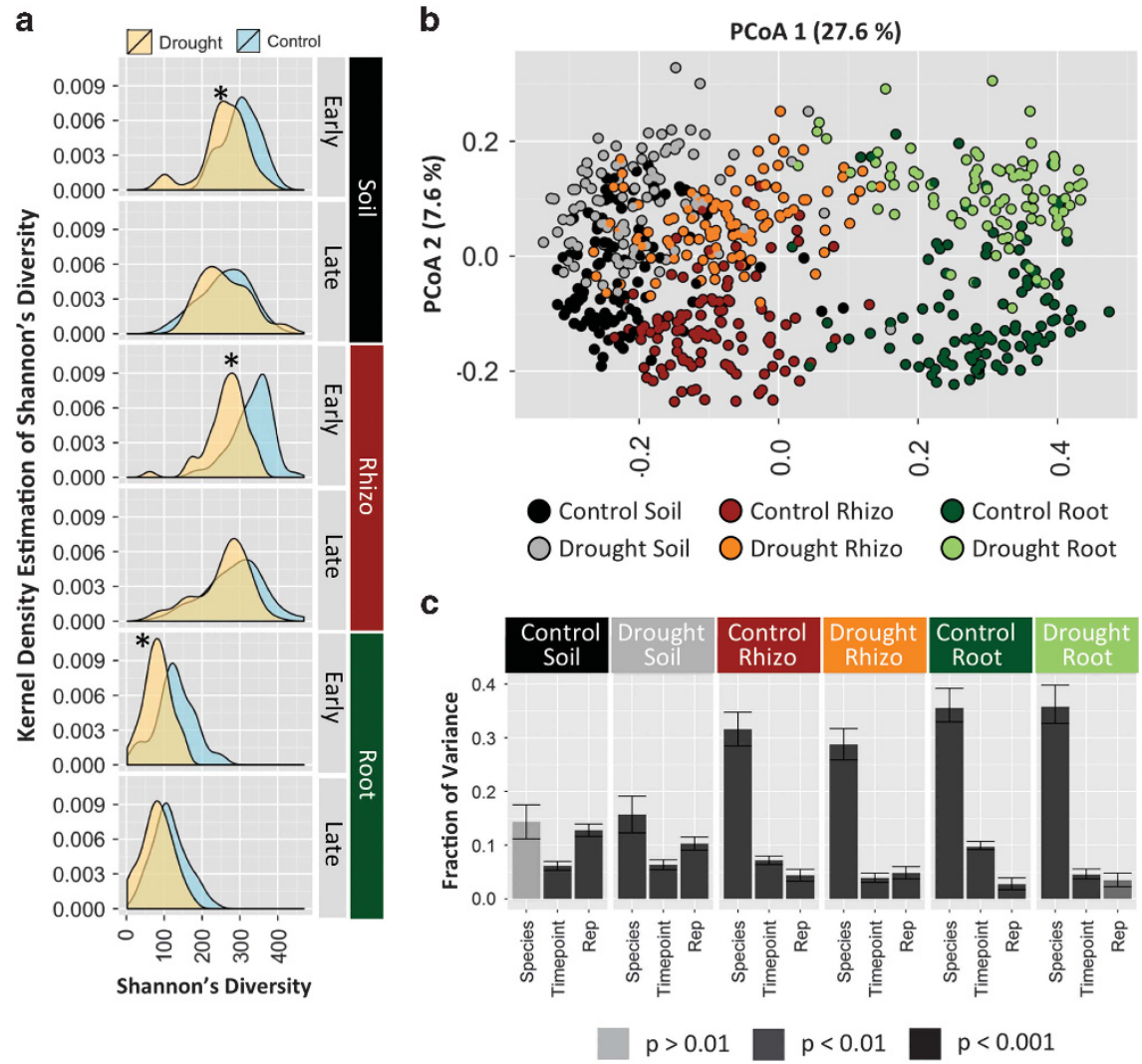

Figure 1 (a) Density plots of Shannon's Diversity in drought (yellow) and control (samples) for each combination of sample type (soil, rhizosphere, root) and time point (early, late). Asterisks indicate drought treatment groups that were significantly different from the respective control group by anlaysis of variance (ANOVA) and Tukey's post hoc tests. (b) Principal coordinate plot for all samples generated using the Bray-Curtis distance; samples are colored for each combination of sample type and treatment. (c) Percent of variance explained by each factor as determined by canonical analysis of principal coordinates (CAPS) using the Bray-Curtis distance for remaining three experimental factors (species, time point and replicate), performed separately for each pairwise combination of sample type and treatment. The y-axis indicates the fraction of variance explained, and the shade of the bar indicates the significance. Error bars indicate a $95 \%$ bootstrap confidence interval around each fraction of variance explained.

plant growth promotion during periods of drought (Casanovas et al., 2002; Marasco et al., 2012), their responsiveness to drought in soils (Bouskill et al., 2013) and their relative abundance within the root microbiome (Shakya et al., 2013; de Souza et al., 2016). By comparison, fungal communities in soils and rhizosphere have been observed to remain relatively similar between drought and control conditions (Barnard et al., 2013; Blazewicz et al., 2014), and archaea are a relatively minor component of the total community in agricultural sites (Taketani et al., 2015). Community analyses were performed using paired-end sequencing of the V3-V4 region of the ribosomal $16 \mathrm{~S}$ rRNA on the Illumina MiSeq platform (Illumina Inc., San Diego, CA, USA), generating 57380917 reads (Supplementary Table S3). These raw sequence data were demultiplexed, trimmed, quality-filtered, clustered and assigned taxonomies, resulting in a reduction to 25694125 read counts, which mapped to 8434 distinct OTUs based on $97 \%$ similarity threshold. After removing low abundance OTUs and normalizing to account for differences in read depth, the data set included 2760 OTUs representing 32 distinct phyla of bacteria.
Bacterial diversity in the grass root microbiome is influenced by drought and host species

To investigate how bacterial $\alpha$-diversity was affected by sample type, time point, species and watering treatment, Shannon's diversity index was calculated for each sample. Analysis of variance revealed that all four factors had significant effects, and that sample type had the greatest effect (Supplementary Table S4). $\alpha$-Diversity decreases significantly from soil to root, as found in similar previous studies (Rascovan et al., 2016), although rhizosphere diversity was not significantly lower than that found in the soil. Within the nine most abundant bacterial classes, $\alpha$-diversity was more affected by sample type for some than others. The class Actinobacteria displayed the most significant decrease in $\alpha$-diversity in roots (Supplementary Figure S3 and Supplementary Table S5), and this was attributed mainly to one genus, Streptomyces, which was greatly enriched in roots relative to soils, representing up to $50 \%$ of all Actinobacteria read counts (Supplementary Figure S4). Of the remaining three factors, treatment had the greatest effect, followed by species and time point. For treatment, median values of Shannon's diversity were lower for drought than 
for control in all pairwise combinations of sample type and time point, although Tukey's post hoc tests indicated that these differences were only significant at early time points (Figure 1a).

To determine which factors most influenced differences in community structure between samples, we investigated $\beta$-diversity through principal coordinate analysis using Bray-Curtis dissimilarities generated for all samples (Figure 1b). Taken together, the first two axes explained $35.4 \%$ of the variance, with the primary axis $(27.6 \%)$ primarily distinguishing samples by sample type and the secondary axis $(7.6 \%)$ by watering treatment (Figure 1b). Principal coordinate analysis based on weighted UniFrac distances (Supplementary Figure S5a) supported similar trends, with the two axes explaining a greater proportion of variance $(37.6 \%$ and $17.4 \%$, respectively). To investigate the amount of variance in the data set attributable to each of our experimental variables, we used canonical analysis of principal coordinates, which allows constrained evaluation for each experimental factor. After separating the data set into groups based on sample type and watering treatment, which are the two most significant factors (Supplementary Table S6a), nearly all remaining factors were significant in all sample groups, but host species explained a relatively large proportion of variance in both rhizosphere and root under both watering treatments (Figure 1c and Supplementary Table S6b). Interestingly, the level of variance explained by host species was not significantly greater in drought than control samples,
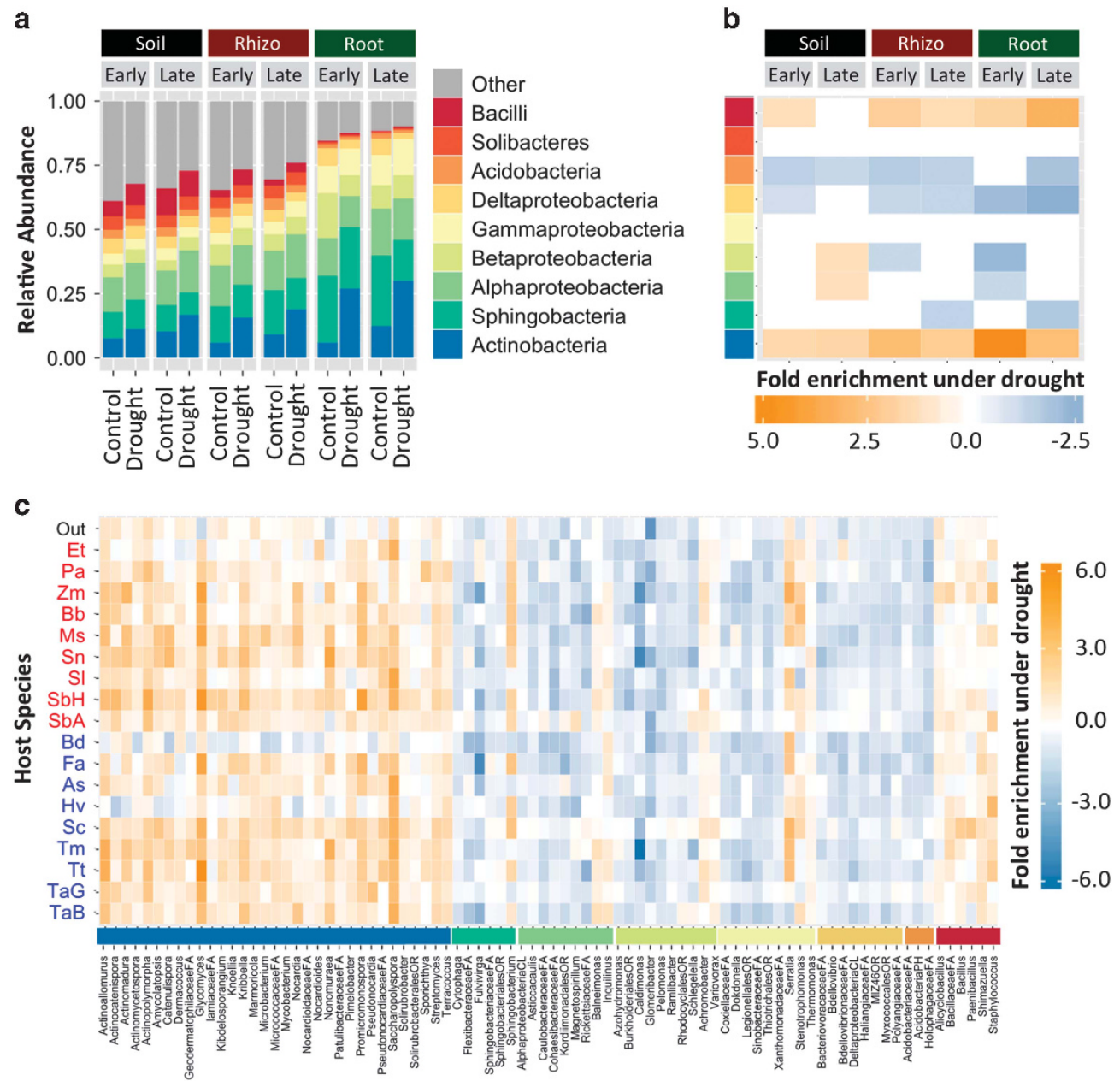

Figure 2 (a) The relative abundance of each of the top nine most abundant bacterial classes for each pairwise combination of sample type, time point and treatment. (b) The fold enrichment under drought of each of the top nine most abundant bacterial classes for each pairwise combination of sample type and time point. (c) Heatmap displaying the fold enrichment under drought (yellow) or control (blue) in roots for all bacterial genera (x-axis) belonging to the top nine most abundant classes and identified as statistically enriched in either drought or control treatments through indicator species analysis. Enrichment is shown independently for each of the 19 host species (y-axis). The class that each genus belongs to is indicated by the colored bar above its name (colors represent the same class as in (a) and (b)). Enrichment is shown independently for each of the 19 host species (y-axis). Abbreviations correspond to host species as follows (Out $=$ Solanum lycopersicum, Et $=$ Eragrostis tef, $\mathrm{Pa}=$ Pennisetum americanum, $\mathrm{Zm}=$ Zea mays, Bb $=$ Bothriochloa bladhii, $\mathrm{Ms}=$ Miscanthus sinensis, $\mathrm{Sn}=$ Sorghastrum nutans, Sl=Sorghum laxiflorum, $\mathrm{SbH}=$ Sorghum bicolor H, SbA=Sorghum bicolor A, $\mathrm{Bd}=$ Brachypodium distachyon, Fa $=$ Festuca arundinaceae, As =Avena sativa, Hv $=$ Hordeum vulgare, Sc $=$ Secale cereale, $\mathrm{Tm}=$ Triticum monococcum, $\mathrm{Tt}=$ Triticum turgidum, $\mathrm{TaG}=$ Triticum aestivum $\mathrm{G}, \mathrm{TaB}=$ Triticum aestivum B). 
contradicting our hypothesis that drought might increase the effect of host species on community composition. Indeed, when using weighted UniFrac distances, the variance attributable to host species was lower under drought than control conditions (Supplementary Figure S5b), suggesting that drought may instead induce conserved shifts in the root and rhizosphere microbiome across our 18 grass hosts. Finally, a smaller experiment to test the effect of drought on fungal communities was performed using fungal-specific ITS2 amplicon sequencing of samples belonging to only the early time point from one replicate block. Through principal coordinate analysis and canonical analysis of principal coordinate analysis, we observed that drought had no significant effect on community composition in any of the three sample types (Supplementary Figure S6), as had been reported recently for comparative analyses of dry and wet soils (Barnard et al., 2013).
Relative abundance shifts indicate enrichment for Actinobacteria under drought

We next investigated changes in relative abundance patterns associated with drought. As has been reported for drought-treated soils and rhizospheres (Blazewicz et al., 2014; Taketani et al., 2016), the most striking change observed was enrichment for Actinobacteria (Figure 2a). Notably, this enrichment was most pronounced in the roots, where Actinobacteria exhibited a 3.1-fold increase, as compared with 2.3- and 1.5-fold enrichment in rhizospheres and soils, respectively (Figure 2b). Enrichment under drought was also observed for class Bacilli, whereas Acidobacteria and Deltaproteobacteria were relatively diminished. To determine if these trends were driven by many or few members of each bacterial class, we investigated enrichment patterns in roots for all bacterial genera identified as indicators of either drought or control treatments through

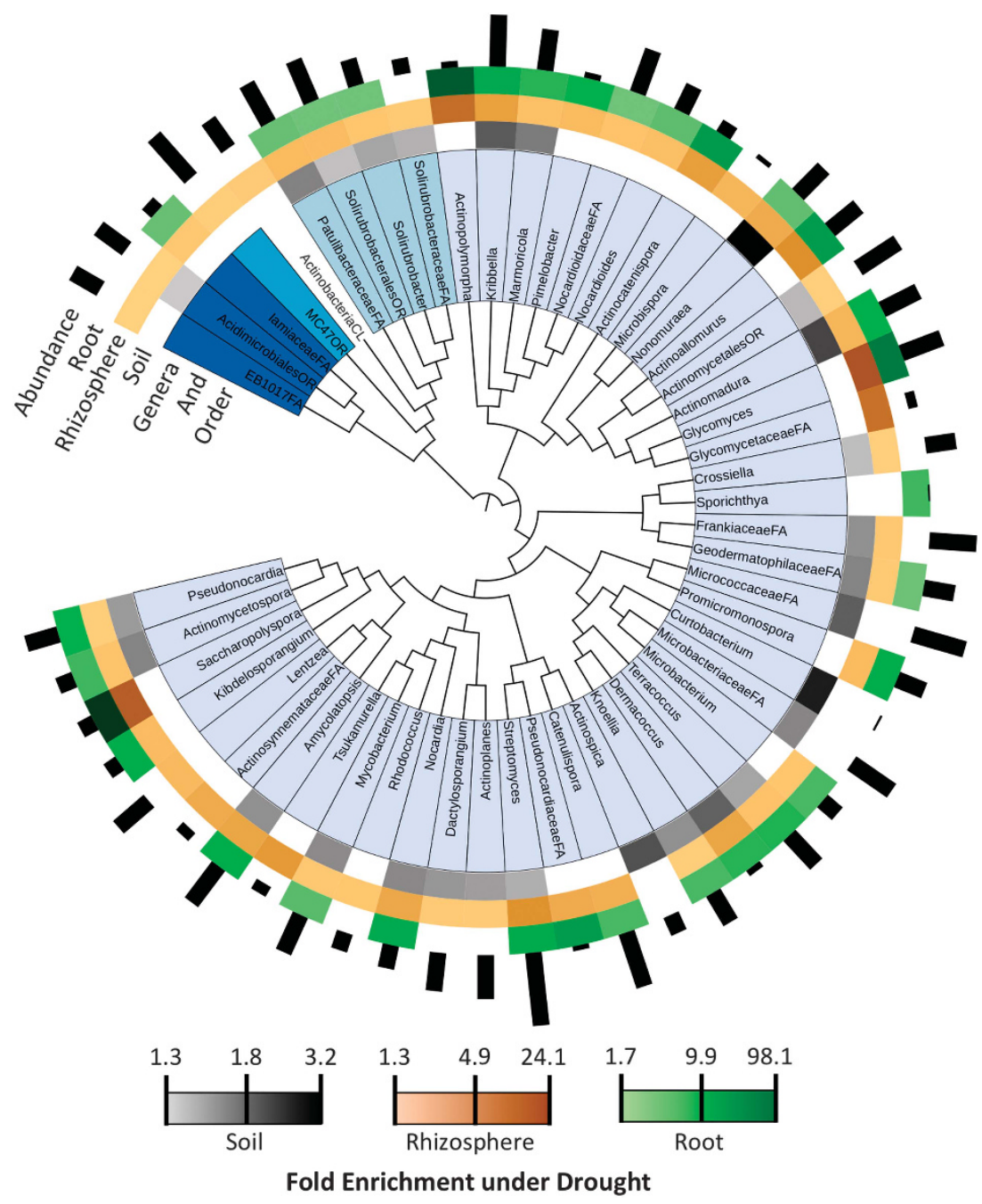

Figure 3 Phylogenetic tree of all Actinobacteria genera identified as indicators of drought treatment in any sample type. The inner ring indicates the five orders these genera belong to; the second, third and fourth innermost rings indicate the degree of $\log _{10}$ fold enrichment in the soil, rhizosphere and root, respectively (legend at bottom). Darker shades of color indicate greater enrichment, whereas white indicates no significant enrichment. The outer ring indicates the $\log _{10}$ abundance of each genus in all samples. 
indicator species analysis (Dufrene and Legendre, 1997) (Figure 2c). All indicator genera belonging to both Actinobacteria (52) and Bacilli (8) were enriched under drought relative to control-in fact, there were no Actinobacteria identified as indicators of the control treatment in any sample type (Supplementary Figure S7). By contrast, most indicator genera belonging to other classes exhibited enrichment under control conditions (Figure 2c). This enrichment pattern was found broadly across all host species, including the non-grass outgroup tomato (Figure 2c and Supplementary Figure S8), suggesting that this phenomenon might be generally true for both monocots and dicots. While the largest effect was a conserved increase in Actinobacteria across all hosts, we did observe that Actinobacteria represented a larger fraction of the drought-treated root microbiome in C4 than in C3 grasses (Supplementary Figure S8), and relative enrichment between drought and control was on average more pronounced in C4 grasses (3.4-fold enrichment in C4 compared with 2.7 in C3). A phylogenetic reconstruction of all indicator Actinobacterial lineages (Figure 3) showed that while 33\% of genera are enriched under drought in all sample types, the majority were specifically enriched in either roots and/or rhizosphere (Saccharopolyspora, Actinopolymorpha, Glycomyces) or exclusively in soils (Kribbella, Curtobacterium, Nonomurea), and that there was no overarching trend with respect to Actinobacterial taxonomy and sample type-specific enrichment patterns. Replication of this experiment with a subset of host species at an additional field site located at the Kearney Agricultural Research station showed similar trends in Actinobacterial enrichment under drought (Supplementary Figures S9a and S10a). The effect was particularly pronounced in the roots, and indeed most Actinobacterial genera enriched under drought at our Albany field site were also enriched at the Kearney field site (Supplementary Figure S10b), with two exceptions (Catellatospora and Virgisporangium), which were enriched under control conditions. However, overlap in the enrichment patterns were not limited to Actinobacteria; Although there were some discrepancies, most bacterial lineages identified as enriched under drought or control in Albany had similar patterns when their respective abundance trends at the Kearney site were examined (Supplementary Figures S9b and S10b).

As some genera of Actinobacteria sporulate under unfavorable environmental conditions (Bergey's, 2010), the relative increase in abundance observed here and in recent studies has been hypothesized to be due to DNA replication and cell division inherent to sporulation (Taketani et al., 2016); if true, the resulting cells would be largely quiescent and perhaps unlikely to affect community function (McCormick and Flärdh, 2012). To test this hypothesis, we documented all Actinobacterial lineages with genus level-classifications enriched under drought in all sample types (32). A literature search indicated that only half of these lineages have been observed to form spores (Supplementary Figure S11). Similarly, a bioinformatic analysis of representative sequenced genomes indicated that only half of these genera contain copies of two genes ( $\operatorname{ssg} B$ and $b l d D$ ) essential to Actinobacterial sporulation (Bush et al., 2015). An alternative explanation for the observed enrichment could be that Actinobacteria do not in fact increase in absolute abundance, but instead other bacterial lineages perish under these conditions, leading to the observed relative increase. To test this hypothesis, we performed quantification of absolute abundance of Actinobacteria in roots under both drought and control conditions using digital droplet PCR (Supplementary Figure S12). The results demonstrate that Actinobacteria exhibit a marked increase in absolute abundance in drought-treated roots for all C3 and C4 grass hosts tested (Supplementary Figure S12). Taken together, the above analyses suggest that neither decreases in the abundances of other taxa nor sporulation are likely to be the only factors responsible for enrichment of Actinobacteria under drought in roots observed here, although further experimental evidence is needed to test how universal these patterns are.

\section{Phylogenetic distance between host species is} correlated with root endosphere community distance We next used the data set to test our hypotheses that host phylogenetic distance is positively correlated with microbiome community distance inside the plant root, and that drought strengthens this correlation. To do so, we first determined plant host distances through sequencing three chloroplast genes, matK/trnK, rbcL and $n d h F$, for each host species and performing phylogenetic analysis on the concatenated sequences (Figure 4a). We used Mantel's tests to compare distances based on host phylogeny and Bray-Curtis dissimilarity (Figure $4 \mathrm{~b}$ ) for each pairwise combination of sample type, time point, treatment and photosynthesis (C3 and C4); the latter segregation was introduced to aid in detection of the correlation between species with a simple nested-monophyletic relationship, as is broadly true of the members chosen here to represent the $\mathrm{C} 3$ and C4 clades. These analyses indicated that root samples generally displayed a significant correlation between host phylogenetic distance and microbiome distance, but that Mantel's $R$-statistic values for rhizosphere samples tended to be smaller and not always significant. Soil microbiome samples showed no significant correlation. Contrary to our hypothesis that environmental stress might induce host-specific shifts in root-associated bacterial communities, at the early time point drought significantly reduced the size of the correlation in both rhizosphere and roots. The early time point is also the time point in which we observe the greatest fold 


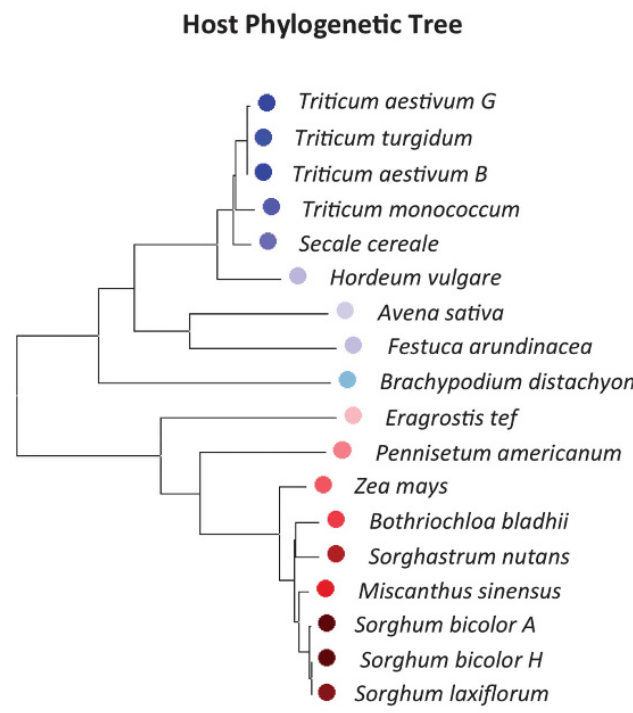

b

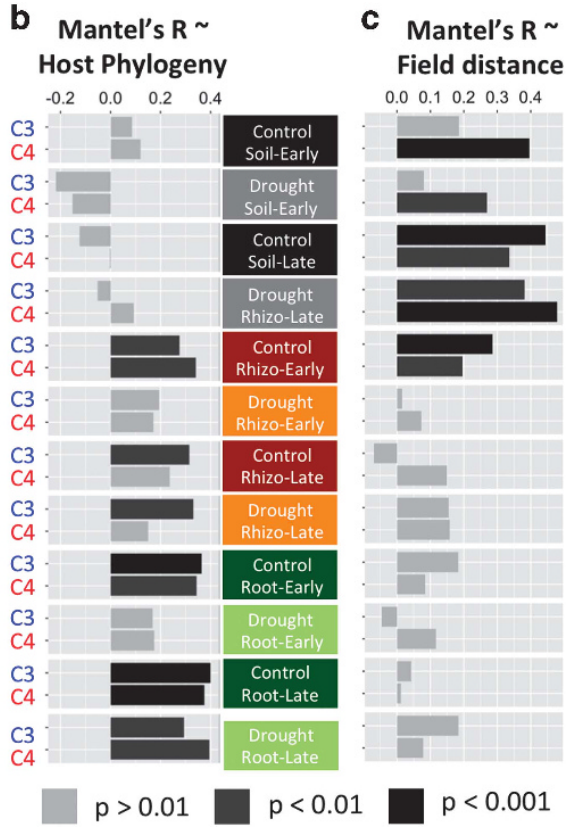

Figure 4 (a) Phylogenetic tree for the 18 grass lineages used in this experiment, constructed from an alignment of three conserved fulllength chloroplast gene sequences ( $r b c L, \operatorname{matK}$, trnK); nodes for C3 grasses are colored in shades of blue, and C4 grasses are colored in shades of red. (b) The Mantel's $R$ coefficient is plotted for pairwise comparisons of host phylogenetic distance and microbiome distance for each pairwise combination of sample type, treatment and time point. To maintain sample evenness across the surveyed phylogenetic breadth of host species, C3 and C4 clades were analyzed separately with the inclusion of a single member from the other clade and the outgroup species. The shade of each bar indicates the significance. (c) The Mantel's $R$ coefficient is plotted for pairwise comparisons of field distance and microbiome distance for each pairwise combination of sample type, treatment and time point. The shade of each bar indicates the significance.

enrichment in Actinobacteria between drought and control across both roots and rhizosphere (Figures 2a and b). However, at the late time point, we observed no significant reduction in the size of the correlation, which could be explained by the emergence of additional and/or stronger host selection phenotypes appearing later in plant development. Interestingly, when a Mantel's test was used to compare pairwise matrices for the microbiome and physical distances between host plants, the opposite pattern was seen, with significant and large correlation in soils, but no correlation observed in rhizosphere and roots (Figure 4c). Taken together, these results confirm our hypothesis that community composition is most highly correlated with host phylogenetic distance within the roots. However, in contrast to our hypothesis that drought would strengthen this correlation, we observed evidence that drought weakens it.

Common taxa in the grass microbiome

Our experimental design, incorporating 18 grass species grown in a single environment, allowed for exploration of the taxa found in common across each of our grass root microbiomes. First, the taxa commonly observed in each individual host species was determined by retaining only those OTUs that were present in at least $30 \%$ of samples and which represent at least $0.01 \%$ of read count abundance for root samples for that host. To obtain the list of taxa found in common across the broader grass root microbiome, we identified those OTUs that were shared between the 18 lists of taxa common to each individual grass host (Supplementary Figure S13). Of these 224 OTUs, which represent $8.1 \%$ of all OTUs and $26.1 \%$ of total read count abundance, all but 6 were also identified as members of the taxa commonly found in tomato, suggesting that the microbes identified here as taxa common to the grass root microbiome are not necessarily specific to grasses but instead represent commonly enriched root endophytes of both monocots and dicots. Pairwise comparisons of individual lists of common taxa for all 19 hosts (Figure 5) indicate a varying degree of similarity (ranging from 42.2 to $73.5 \%$ overlap). Interestingly, we observed that the three species of sorghum had larger sets of commonly observed taxa than any other host. Notably, two of these hosts (Sorghum bicolor $H$ and Sorghum laxiflorum) also had the lowest median overlap with the lists of taxa commonly observed in the other hosts (55.8 and 58.3\%), including the outgroup tomato $(59.4 \%)$. Taken together, these results suggest that while a collective set of common root taxa exists for grass species, these microbes represent a small fraction of all root OTUs $(6 \%)$ and are likely generalist colonizers of the roots of most plants. However, as field site and soil type can have a large effect on overall 

a

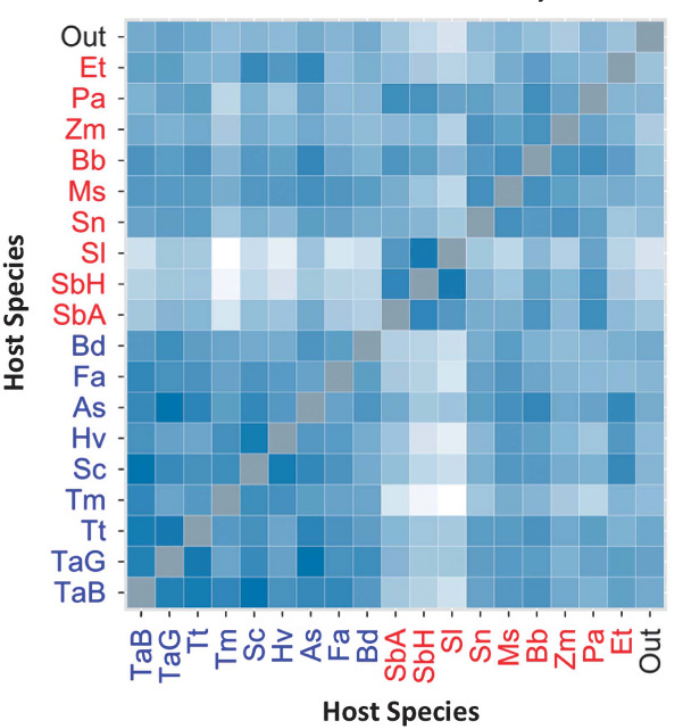

b

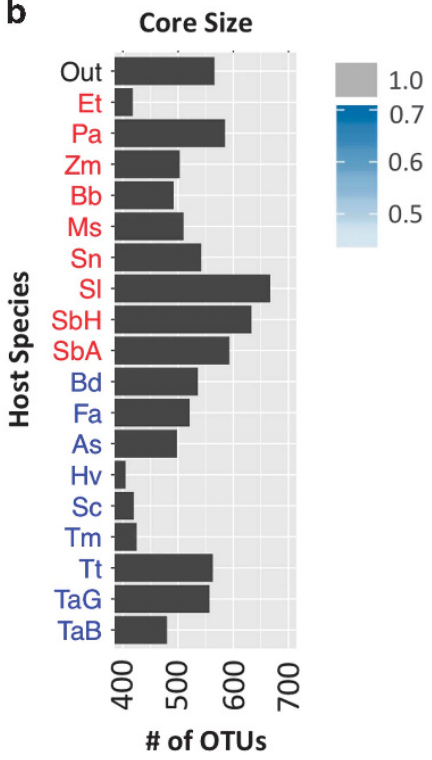

Figure 5 (a) Heatmap displaying the percent of overlap between the taxa common to the root microbiome of each host with every other host; lighter shades of blue represent less overlap, while darker shades represent more. Host species identifiers are the first letter of the genus and species (e.g. Zm for Zea mays). (b) The size of the list of common taxa as the number of OTUs identified as member of each list for each of the 19 host species.

microbiome composition, further work will be needed to determine the reproducibility of these results in other systems.

\section{Discussion}

Cereal host root microbiome composition correlates with host phylogenetic distance

A wide range of host and environmental factors have been shown to influence plant microbiome composition (Berg and Smalla, 2009; Gaiero et al., 2013). Using canonical analysis of principal coordinates analysis, we found that sample type, host species and watering treatment were the three most important sources of compositional variance. Sample type has been implicated as the largest source of variation in multicompartment data sets (Lundberg et al., 2012; Marasco et al., 2012; Bulgarelli et al., 2015; Cherif et al., 2015; Edwards et al., 2015; ColemanDerr et al., 2016; Fonseca-García et al., 2016). Previous studies investigating the effect of host genetics have tended to focus on varieties of a single given host species, such as barley (Bulgarelli et al., 2015), maize (Peiffer et al., 2013) or agave (Desgarennes et al., 2014; Coleman-Derr et al., 2016), or on a few distinct species (el Zahar Haichar et al., 2008; Bouffaud et al., 2014; Schlaeppi et al., 2014). In these studies, species effects tended to be nonsignificant (Schlaeppi et al., 2014) or small (Lundberg et al., 2012; Peiffer et al., 2013; Fonseca-García et al., 2016). By comparison, we found that host species was highly significant and explained $\sim 19.4$ and $23.4 \%$ of variance within the rhizosphere and root endosphere. The species effect size observed in our study as compared with the relatively low values observed in previous studies suggests that the influence of host species on the root microbiome should be considered in the context of the working phylogenetic framework, and a broader array of considered species will accordingly produce a greater host effect. Differences in the developmental timelines between species may have introduced variability with respect to developmental stage at the time of harvest between species; however, based on field observations for flowering time, the time points we selected broadly represented pre- and postflowering stages for nearly all species. In contrast to our hypothesis that drought might increase the differences in root community composition between different hosts, we observed that the effect of host species was not substantially different between drought and control when using Bray-Curtis distances, and was marginally reduced when using weighted UniFrac distances. These results are consistent with the large shift in Actinobacteria we observe in roots, which is broadly conserved across all hosts we examined. Other environmental stresses may provoke similar trends in conserved enrichment patterns across hosts, whether in Actinobacteria or other bacterial taxa, and will need to be investigated.

While previous studies have confirmed the role of genotype in determining microbiome composition, very few have tested the correlation between host phylogenetic and microbiome distances. In examining four Brassicaceae members, microbiota diversification was not consistent with host distance (Schlaeppi et al., 2014); similarly, two studies of maize inbred lines (Bouffaud et al., 2012; Peiffer 
et al., 2013) found that differences in rhizobacterial communities did not correlate with host genetic distance. However, these studies may have been hampered by low sample size and close relatedness of host species. A study using five maize varietals and two additional Poaceae members (Bouffaud et al., 2014) did detect a significant correlation between plant phylogenetic distance and microbiome distance in rhizospheres, but did not investigate root endospheres. In this study, we expand on previous results by: (1) incorporating many distinct species from both C3 and C4 grasses; (2) examining the relative effect size in the rhizosphere and root endosphere; and (3) investigating how environmental stress might perturb the relationship between host phylogeny and microbiome composition. Our data confirm our hypothesis of a positive correlation between host phylogenetic and microbiome distance that is strongest in roots, weaker in the rhizosphere and nonexistent in the surrounding soil, indicating that host-selective forces on the microbiome grow in magnitude with increased plant-microbe intimacy, which has been recently demonstrated in two studies (Beckers et al., 2017; Samad et al., 2017). We also observed that drought generally decreased the effect size and significance of the Mantel's statistic, most notably at the early time point, contradicting our hypothesis that drought strengthens the correlation between host phylogeny and microbiome distance due to host species-specific drought response mechanisms. Instead, these results are consistent with the idea that conserved plant responses to drought, especially early in development (such as increased ABA production), have larger effects on microbiome composition than individual and species-specific adaptations. Additionally, the observation that in the late time point drought provokes a less similar, and therefore a more host-specific, microbial community across hosts, as indicated by the relatively larger size and significance of the Mantel's correlation, is evidence that perhaps host specificity due to species-specific drought responses may take time to accumulate. Phylogenetic distance is not the only factor that differentiates our selected grasses: for example, root morphology can vary significantly between different grasses (Rich and Watt, 2013) and could have affected the microbiome composition. To account for this factor, plant roots were harvested to a consistent rooting depth $(25 \mathrm{~cm})$ for all species, athough it is possible that root morphology or other species-specific root characteristics that do not necessarily correlate with host phylogenetic relatedness may represent compounding factors in our analysis of the host effect.

\section{Actinobacteria enrichment under drought}

To our knowledge this represents the first major investigation of the role of drought in determining root endosphere microbiome composition. Here we identified watering treatment as a significant factor in determining community composition within root communities; drought was found to explain $8.8 \%$ and $9.9 \%$ of variance within the root and rhizosphere, as compared to $5.6 \%$ within soils. While drought induced many changes in microbiome composition, the most significant at the class level was an increase of Actinobacteria. While enrichment of Actinobacteria under drought has been reported in soils (Hayden et al., 2012; Bouskill et al., 2013, 2016) and rhizosphere (Nessner Kavamura et al., 2013; Taketani et al., 2016), enrichment has not been reported in the root endosphere, nor have enrichment trends been compared between these distinct microhabitats. Our observation that enrichment of Actinobacteria under drought is significantly higher in the root endosphere compared with either rhizosphere or bulk soil suggests that while the Actinobacterial enrichment under drought is not unique to roots, it is enhanced by them. Indeed, 24 genera, including Saccharopolyspora, Glycomyces and Actinopolymorpha, were exclusively enriched in roots and rhizosphere and not in surrounding soil. The observed enrichment in Actinobacteria under drought treatment within roots was found in two field sites, suggesting that these results may be generalizable not only across different plant hosts but also across distinct environments as well. Additionally, while levels of enrichment for individual Actinobacterial lineages were largely consistent across all hosts, there were notable exceptions. For example, an Actinobacteria genus with the most significant enrichment under drought in grass roots (20.3-fold), Glycomyces, had the opposite pattern in tomato. Further comparative genomic analyses and functional studies using these lineages that exhibit sample type- and host-specific enrichment patterns might help decipher underlying mechanisms involved in this process.

The general enrichment of nearly all Actinobacteria under drought suggests that the force driving this enrichment is related to one or more conserved properties of the Actinobacterial lineage. For example, among bacteria, they are highly tolerant of life in arid environments (Bull and Asenjo, 2013; Pearce et al., 2013; Stevenson and Hallsworth, 2014; Cherif et al., 2015) where other bacterial lineages have difficulty surviving, owing in part to their ability to form spores. Our observation that only about half of drought-enriched Actinobacteria in our study are likely capable of forming spores suggests that sporulation is not fully responsible for their prevalence under drought observed here. Additionally, the two Actinobacteria genera observed to be enriched under control conditions in the Kearney data set are both reported to be capable of forming spores, suggesting that the ability to form spores does not necessarily lead to enrichment under drought conditions (Bergey's, 2010); however, the evidence presented in this study is based on reports in the literature and bioinformatic analyses, and further experimental 
evidence is needed to determine whether this pattern holds true for other lineages and in other environments and hosts.

Alternatively, the strong enrichment for Actinobacteria within plant-associated samples could be the result of direct or indirect effects due to droughtinduced changes in plant root traits, exudation patterns or changes in niche opportunities on and inside the root surface. One putative mechanism for this selection could include shifts in cell wall biochemistry, as plants are known to modulate cell wall components in response to drought (Gall et al., 2015) and as Actinobacteria are capable of responding to and using some of these compounds that may be a part of their own drought response (Pold et al., 2016). Whether this selection is ultimately beneficial for the host remains unclear. Many Actinobacteria, particularly members of order Actinomycetales, are known saprophytes (Solans, 2007) with the capability to degrade relatively recalcitrant plant polymers, such as lignin and suberin (Komeil et al., 2013; de Gonzalo et al., 2016). However, three droughttolerant strains of the Actinobacteria genus Streptomyces were shown to enhance wheat seedling vigor in water-stressed soils (Yandigeri et al., 2012); additionally, Actinobacteria were reported to alleviate drought stress in peppers (Marasco et al., 2012). The highly conserved nature of this enrichment across both diverse hosts and microbes suggests to us that this is an ancient and potentially mutually beneficial relationship, although further molecular and biochemical analyses will be necessary to explore this hypotheses.

\section{Common taxa in the grass root microbiome}

Interestingly, within the 18 grasses tested, the sorghum species exhibited larger and more distinct sets of commonly observed taxa compared with the other hosts. In addition to their remarkable drought tolerance, sorghum plants are known to exude novel secondary metabolites from their roots (Dayan et al., 2010). One such product, the terpenoid sorgoleone, has been shown to have significant allelopathic properties (Uddin et al., 2014), although its effect on the root microbiome has yet to be tested. Despite host-specific differences, all cereals in our study shared a subset of their common root OTUs; it is interesting that more than $95 \%$ of these were also detected as part of the common taxa found in tomato, which suggests that these microbes may be generalist colonizers of the plant root. It should be noted that criteria used to define the shared microbiome have a large effect on the specific taxa that are identified, and that the choices used in this analysis were intentionally permissive to select a broader list of shared microbes. One caveat to our observations for the common taxa microbiome, and indeed the root microbiome generally, is that the microbiome is heavily influenced by the local soil environment from which samples were collected; even genotypically identical grasses may have strikingly distinct root microbiota patterns when grown in different environments (Lundberg et al., 2012; Rascovan et al., 2016). More work will be needed to determine if the patterns observed here are found across a broad range of other soil types and locales.

\section{Conclusion}

Drought is an important selective pressure that may drive plant and bacterial evolutionary responses (terHorst et al., 2014). The influence of drought on bacterial communities in soils is increasingly recognized (Sheik et al., 2011; Pold et al., 2016); our study adds to this emerging field by showing that drought significantly impacts the composition and diversity of the plant root microbiome, and that recently observed enrichments in Actinobacteria under drought are in fact more pronounced in root endosphere than in the surrounding soils. Additionally, our study provides evidence that while different plant hosts show selection for specific root bacterial communities in a manner correlated with their evolutionary histories, drought in fact provokes a relatively conserved response across a broad range of plant species. Our research suggests that previously hypothesized causes for this enrichment in soils, including sporulation and relative fitness in dry environments, are unlikely to fully explain the enrichment observed here in roots. Further research aimed at understanding the causes and consequences for this highly conserved relationship between an entire class of bacteria and many species of plants will be important for improving our understanding of the role that environmental parameters have in shaping plant-bacterial interactions.

\section{Conflict of Interest}

The authors declare no conflict of interest.

\section{Acknowledgements}

We thank Siwen Deng, Heidi Wipf, Melissa Shinfuku and Janki Desai for assistance in the field, Melissa Shinfuku for assistance with DNA extractions, Will Iles for assistance with phylogenetic methods and Sheila McCormick, Steve Lindow and Susannah Tringe for constructive comments on the manuscript. This work was funded by the USDAARS (CRIS 2030-21430-008-00D).

\section{References}

Aleklett K, Leff JW, Fierer N, Hart M. (2015). Wild plant species growing closely connected in a subalpine meadow host distinct root-associated bacterial communities. PeerJ 3: e804.

Bacchetti De Gregoris T, Aldred N, Clare AS, Burgess JG. (2011). Improvement of phylum- and class-specific 
primers for real-time PCR quantification of bacterial taxa. J Microbiol Methods 86: 351-356.

Badri DV, Chaparro JM, Zhang R, Shen Q, Vivanco JM. (2013). Application of natural blends of phytochemicals derived from the root exudates of arabidopsis to the soil reveal that phenolic-related compounds predominantly modulate the soil microbiome. J Biol Chem 288: 4502-4512.

Barnard RL, Osborne CA, Firestone MK. (2013). Responses of soil bacterial and fungal communities to extreme desiccation and rewetting. ISME J 7: 2229-2241.

Beckers B, Op De Beeck M, Weyens N, Boerjan W, Vangronsveld J. (2017). Structural variability and niche differentiation in the rhizosphere and endosphere bacterial microbiome of field-grown poplar trees. Microbiome 5: 25.

Berendsen RL, Pieterse CMJ, Bakker PAHM. (2012). The rhizosphere microbiome and plant health. Trends Plant Sci 17: 478-486.

Berg G, Grube M, Schloter M, Smalla K. (2014). Unraveling the plant microbiome: looking back and future perspectives. Front Microbiol 5: 148

Berg G, Rybakova D, Grube M, Köberl M. (2016). The plant microbiome explored: implications for experimental botany. J Exp Bot 67: 995-1002.

Berg G, Smalla K. (2009). Plant species and soil type cooperatively shape the structure and function of microbial communities in the rhizosphere: plant species, soil type and rhizosphere communities. FEMS Microbiol Ecol 68: 1-13.

Bergey's MO. (2010). The Bacteroidetes, Spirochaetes, Tenericutes (Mollicutes), Acidobacteria, Fibrobacteres, Fusobacteria, Dictyoglomi, Gemmatimonadetes, Lentisphaerae, Verrucomicrobia, Chlamydiae, and Planctomycetes. Available at: http://www.academia.edu/ download/44794212/Noel_R._Krieg_Wolfgang_Lud wig_William_B._WhitmaBookZZ.org.pdf (last accessed 30 December 2016).

Blazewicz SJ, Schwartz E, Firestone MK. (2014). Growth and death of bacteria and fungi underlie rainfallinduced carbon dioxide pulses from seasonally dried soil. Ecology 95: 1162-1172.

Bouffaud M-L, Kyselková M, Gouesnard B, Grundmann G, Muller D, MoëNne-Loccoz Y. (2012). Is diversification history of maize influencing selection of soil bacteria by roots?: Maize diversification and root bacteria. Mol Ecol 21: 195-206.

Bouffaud M-L, Poirier M-A, Muller D, Moënne-Loccoz Y. (2014). Root microbiome relates to plant host evolution in maize and other Poaceae: Poaceae evolution and root bacteria. Environ Microbiol 16: 2804-2814.

Bouskill NJ, Lim HC, Borglin S, Salve R, Wood TE, Silver WL et al. (2013). Pre-exposure to drought increases the resistance of tropical forest soil bacterial communities to extended drought. ISME J 7: 384-394.

Bouskill NJ, Wood TE, Baran R, Ye Z, Bowen BP, Lim H et al. (2016). Belowground response to drought in a tropical forest soil. I. Changes in microbial functional potential and metabolism. Front Microbiol 7: 525.

Brusetti L, Francia P, Bertolini C, Pagliuca A, Borin S, Sorlini C et al. (2005). Bacterial communities associated with the rhizosphere of transgenic Bt 176 maize (Zea mays) and its non transgenic counterpart. Plant Soil 266: 11-21.

Bulgarelli D, Garrido-Oter R, Münch PC, Weiman A, Dröge J, Pan Y et al. (2015). Structure and function of the bacterial root microbiota in wild and domesticated barley. Cell Host Microbe 17: 392-403.

Bull AT, Asenjo JA. (2013). Microbiology of hyper-arid environments: recent insights from the Atacama Desert, Chile. Antonie Van Leeuwenhoek 103: 1173-1179.

Bush MJ, Tschowri N, Schlimpert S, Flärdh K, Buttner MJ. (2015). c-di-GMP signalling and the regulation of developmental transitions in streptomycetes. Nat Rev Microbiol 13: 749-760.

Casanovas EM, Barassi CA, Sueldo RJ. (2002). Azospirillum inoculation mitigates water stress effects in maize seedlings. Cereal Res Commun 30: 343-350.

Cavaglieri L, Orlando J, Etcheverry M. (2009). Rhizosphere microbial community structure at different maize plant growth stages and root locations. Microbiol Res 164: 391-399.

Chaparro JM, Badri DV, Bakker MG, Sugiyama A, Manter DK, Vivanco JM. (2013). Root exudation of phytochemicals in arabidopsis follows specific patterns that are developmentally programmed and correlate with soil microbial functions. PLoS One 8: e55731.

Cherif H, Marasco R, Rolli E, Ferjani R, Fusi M, Soussi A et al. (2015). Oasis desert farming selects environmentspecific date palm root endophytic communities and cultivable bacteria that promote resistance to drought: Oasis palm endophytes promote drought resistance. Environ Microbiol Rep 7: 668-678.

Coleman-Derr D, Desgarennes D, Fonseca-Garcia C, Gross S, Clingenpeel S, Woyke T et al. (2016). Plant compartment and biogeography affect microbiome composition in cultivated and native Agave species. New Phytol 209: 798-811.

Dayan FE, Rimando AM, Pan Z, Baerson SR, Gimsing AL, Duke SO. (2010). Sorgoleone. Phytochemistry 71: 1032-1039.

de Gonzalo G, Colpa DI, Habib MHM, Fraaije MW. (2016). Bacterial enzymes involved in lignin degradation. J Biotechnol 236: 110-119.

Desgarennes D, Garrido E, Torres-Gomez MJ, Peña-Cabriales JJ, Partida-Martinez LP. (2014). Diazotrophic potential among bacterial communities associated with wild and cultivated Agave species. FEMS Microbiol ECol 90: 844-857.

Dombrowski N, Schlaeppi K, Agler MT, Hacquard S, Kemen E, Garrido-Oter R et al. (2017). Root microbiota dynamics of perennial Arabis alpina are dependent on soil residence time but independent of flowering time. ISME J 11: 43-55.

Dufrene M, Legendre P. (1997). Species assemblages and indicator species: the need for a flexible asymmetrical approach. Ecol Monogr 67: 345-366.

Edwards J, Johnson C, Santos-Medellín C, Lurie E, Podishetty NK, Bhatnagar S et al. (2015). Structure, variation, and assembly of the root-associated microbiomes of rice. Proc Natl Acad Sci USA 112: E911-E920.

Fang Y, Xiong L. (2015). General mechanisms of drought response and their application in drought resistance improvement in plants. Cell Mol Life Sci 72: 673-689.

Fierer N, Schimel JP, Holden PA. (2003). Variations in microbial community composition through two soil depth profiles. Soil Biol Biochem 35: 167-176.

Fonseca-García C, Coleman-Derr D, Garrido E, Visel A, Tringe SG, Partida-Martínez LP. (2016). The Cacti microbiome: interplay between habitat-filtering and host-specificity. Front Microbiol 7: 150. 
Gaiero JR, McCall CA, Thompson KA, Day NJ, Best AS, Dunfield KE. (2013). Inside the root microbiome: bacterial root endophytes and plant growth promotion. Am J Bot 100: 1738-1750.

Gall H, Philippe F, Domon J-M, Gillet F, Pelloux J, Rayon C. (2015). Cell wall metabolism in response to abiotic stress. Plants 4: 112-166.

Ghotbi-Ravandi AA, Shahbazi M, Shariati M, Mulo P. (2014). Effects of mild and severe drought stress on photosynthetic efficiency in tolerant and susceptible barley (Hordeum vulgare L.) genotypes. J Agron Crop Sci 200: 403-415.

Grass Phylogeny Working Group II. (2012). New grass phylogeny resolves deep evolutionary relationships and discovers C4 origins. New Phytol 193: 304-312.

Hayden HL, Mele PM, Bougoure DS, Allan CY, Norng S, Piceno YM et al. (2012). Changes in the microbial community structure of bacteria, archaea and fungi in response to elevated $\mathrm{CO}_{2}$ and warming in an Australian native grassland soil: climate change effects on microbial communities. Environ Microbiol 14: 3081-3096.

Komeil D, Simao-Beaunoir A-M, Beaulieu C. (2013). Detection of potential suberinase-encoding genes in Streptomyces scabiei strains and other actinobacteria. Can J Microbiol 59: 294-303.

Kuske CR, Ticknor LO, Miller ME, Dunbar JM, Davis JA, Barns SM et al. (2002). Comparison of soil bacterial communities in rhizospheres of three plant species and the interspaces in an arid grassland. Appl Environ Microbiol 68: 1854-1863.

Lesk C, Rowhani P, Ramankutty N. (2016). Influence of extreme weather disasters on global crop production. Nature 529: 84-87.

Lundberg DS, Lebeis SL, Paredes SH, Yourstone S, Gehring J, Malfatti S et al. (2012). Defining the core Arabidopsis thaliana root microbiome. Nature 488: 86-90.

Marasco R, Rolli E, Ettoumi B, Vigani G, Mapelli F, Borin S et al. (2012). A drought resistance-promoting microbiome is selected by root system under desert farming. PLoS One 7: e48479.

McCormick JR, Flärdh K. (2012). Signals and regulators that govern Streptomyces development. FEMS Microbiol Rev 36: 206-231.

Nessner Kavamura V, Taketani RG, Lançoni MD, Andreote FD, Mendes R, Soares de Melo I. (2013). Water regime influences bulk soil and rhizosphere of cereus jamacaru bacterial communities in the Brazilian Caatinga biome. PLoS One 8: e73606.

Nezhadahmadi A, Prodhan ZH, Faruq G. (2013). Drought tolerance in wheat. Sci World J 2013: 1-12.

Parker MA, Spoerke JM. (1998). Geographic structure of lineage associations in a plant-bacterial mutualism. $J$ Evol Biol 11: 549-562.

Pearce D, Hodgson D, Thorne M, Burns G, Cockell C. (2013). Preliminary analysis of life within a former Subglacial Lake Sediment in Antarctica. Diversity 5: 680-702

Peiffer JA, Spor A, Koren O, Jin Z, Tringe SG, Dangl JL et al. (2013). Diversity and heritability of the maize rhizosphere microbiome under field conditions. Proc Natl Acad Sci USA 110: 6548-6553.

Philippot L, Raaijmakers JM, Lemanceau P, van der Putten WH. (2013). Going back to the roots: the microbial ecology of the rhizosphere. Nat Rev Microbiol 11: 789-799.
Pold G, Billings AF, Blanchard JL, Burkhardt DB, Frey SD, Melillo JM et al. (2016). Long-term warming alters carbohydrate degradation potential in temperate forest soils. Appl Environ Microbiol 82: 6518-6530.

Quast C, Pruesse E, Yilmaz P, Gerken J, Schweer T, Yarza P et al. (2013). The SILVA ribosomal RNA gene database project: improved data processing and webbased tools. Nucleic Acids Res 41: D590-D596.

Rascovan N, Carbonetto B, Perrig D, Díaz M, Canciani W, Abalo $\mathrm{M}$ et al. (2016). Integrated analysis of root microbiomes of soybean and wheat from agricultural fields. Sci Rep 6: 28084.

Rich SM, Watt M. (2013). Soil conditions and cereal root system architecture: review and considerations for linking Darwin and Weaver. J Exp Bot 64: 1193-1208.

Sabadin PK, Malosetti M, Boer MP, Tardin FD, Santos FG, Guimarães CT et al. (2012). Studying the genetic basis of drought tolerance in sorghum by managed stress trials and adjustments for phenological and plant height differences. Theor Appl Genet 124: 1389-1402.

Samad A, Trognitz F, Compant S, Antonielli L, Sessitsch A. (2017). Shared and host-specific microbiome diversity and functioning of grapevine and accompanying weed plants: microbial communities associated with grapevine and vineyard weeds. Environ Microbiol 19: 1407-1424.

Schlaeppi K, Dombrowski N, Oter RG, Ver Loren van Themaat E, Schulze-Lefert P. (2014). Quantitative divergence of the bacterial root microbiota in Arabidopsis thaliana relatives. Proc Natl Acad Sci USA 111: 585-592.

Shakya M, Gottel N, Castro H, Yang ZK, Gunter L, Labbé J et al. (2013). A multifactor analysis of fungal and bacterial community structure in the root microbiome of mature populus deltoides trees. PLoS One 8: e76382.

Sheik CS, Beasley WH, Elshahed MS, Zhou X, Luo Y, Krumholz LR. (2011). Effect of warming and drought on grassland microbial communities. ISME $J$ 5: 1692-1700.

Shi S, Richardson AE, O’Callaghan M, DeAngelis KM, Jones EE, Stewart A et al. (2011). Effects of selected root exudate components on soil bacterial communities: root exudate components and soil microbial communities. FEMS Microbiol Ecol 77: 600-610.

Solans M. (2007). Discaria trinervis-Frankia symbiosis promotion by saprophytic actinomycetes. J Basic Microbiol 47: 243-250.

de Souza RSC, Okura VK, Armanhi JSL, Jorrín B, Lozano N, da Silva MJ et al. (2016). Unlocking the bacterial and fungal communities assemblages of sugarcane microbiome. Sci Rep 6: 28774.

Stevenson A, Hallsworth JE. (2014). Water and temperature relations of soil Actinobacteria: water and temperature relations of Actinobacteria. Environ Microbiol Rep 6: $744-755$.

Takahashi S, Tomita J, Nishioka K, Hisada T, Nishijima M. (2014). Development of a prokaryotic universal primer for simultaneous analysis of bacteria and archaea using next-generation sequencing. PLoS One 9: e105592.

Taketani RG, Kavamura VN, Mendes R, Melo IS. (2015). Functional congruence of rhizosphere microbial communities associated to leguminous tree from Brazilian semiarid region: functional congruence in semiarid. Environ Microbiol Rep 7: 95-101.

Taketani RG, Lançoni MD, Kavamura VN, Durrer A, Andreote FD, Melo IS. (2016). Dry season constrains 
bacterial phylogenetic diversity in a semi-arid rhizosphere system. Microb Ecol 73: 153-161.

terHorst CP, Lennon JT, Lau JA. (2014). The relative importance of rapid evolution for plant-microbe interactions depends on ecological context. Proc $R$ Soc Ser B 281: 20140028-20140028.

Uddin MR, Park SU, Dayan FE, Pyon JY. (2014). Herbicidal activity of formulated sorgoleone, a natural product of sorghum root exudate: herbicidal effect of formulated sorgoleone. Pest Manag Sci 70: 252-257.

Ushio M, Wagai R, Balser TC, Kitayama K. (2008). Variations in the soil microbial community composition of a tropical montane forest ecosystem: does tree species matter? Soil Biol Biochem 40: 2699-2702.

Yandigeri MS, Meena KK, Singh D, Malviya N, Singh DP, Solanki MK et al. (2012). Drought-tolerant endophytic actinobacteria promote growth of wheat (Triticum aestivum) under water stress conditions. Plant Growth Regul 68: 411-420.

Yang Y-W, Chen M-K, Yang B-Y, Huang X-J, Zhang X-R, He L-Q et al. (2015). Use of $16 \mathrm{~S}$ rRNA gene-targeted group-specific primers for real-time PCR analysis of predominant bacteria in mouse feces. Appl Environ Microbiol 81: 6749-6756.

el Zahar Haichar F, Marol C, Berge O, Rangel-Castro JI, Prosser JI, Balesdent J et al. (2008). Plant host habitat and root exudates shape soil bacterial community structure. ISME J 2: 1221-1230.

(c) (1) (2) This work is licensed under a Creative Commons Attribution-NonCommercialShareAlike 4.0 International License. The images or other third party material in this article are included in the article's Creative Commons license, unless indicated otherwise in the credit line; if the material is not included under the Creative Commons license, users will need to obtain permission from the license holder to reproduce the material. To view a copy of this license, visit http://creativecommons.org/ licenses/by-nc-sa/4.0/

(C) The Author(s) 2017

Supplementary Information accompanies this paper on The ISME Journal website (http://www.nature.com/ismej) 